\title{
Lateral gene transfer, rearrangement, reconciliation
}

\author{
Murray Patterson ${ }^{1,2,3^{*}}$, Gergely Szöllösi ${ }^{2,4}$, Vincent Daubin², Eric Tannier ${ }^{1,2^{*}}$ \\ From Eleventh Annual Research in Computational Molecular Biology (RECOMB) Satellite Workshop on \\ Comparative Genomics \\ Lyon, France. 17-19 October 2013
}

\begin{abstract}
Background: Models of ancestral gene order reconstruction have progressively integrated different evolutionary patterns and processes such as unequal gene content, gene duplications, and implicitly sequence evolution via reconciled gene trees. These models have so far ignored lateral gene transfer, even though in unicellular organisms it can have an important confounding effect, and can be a rich source of information on the function of genes through the detection of transfers of clusters of genes.

Result: We report an algorithm together with its implementation, DeCoLT, that reconstructs ancestral genome organization based on reconciled gene trees which summarize information on sequence evolution, gene origination, duplication, loss, and lateral transfer. DeCoLT optimizes in polynomial time on the number of rearrangements, computed as the number of gains and breakages of adjacencies between pairs of genes. We apply DeCoLT to 1099 gene families from 36 cyanobacteria genomes.
\end{abstract}

Conclusion: DeCoLT is able to reconstruct adjacencies in 35 ancestral bacterial genomes with a thousand gene families in a few hours, and detects clusters of co-transferred genes. DeCoLT may also be used with any relationship between genes instead of adjacencies, to reconstruct ancestral interactions, functions or complexes.

Availability: http://pbil.univ-lyon1.fr/software/DeCoLT/

\section{Introduction}

The evolution of genomes can be explored at two different scales. At the chromosome level, rearrangements have been studied from the 1930's [1,2], and have progressively incorporated the possibility of unequal gene content, gene duplications and gene losses [3,4]. Later, but largely independently, in the 1960's, the evolution of genomic sequences began to be modeled [5,6], and has more recently been extended to include the duplication, loss and the lateral transfer of genes via the reconciliation of gene trees with a species tree [7-11]. These two scales have only met on a few occasions through the integration of phylogenies and rearrangements [12,13], using reconciled phylogenies to account for the duplication and loss of genes. Here, we build on these ideas and reconstruct

\footnotetext{
* Correspondence: Murray.Patterson@cwi.nl; Eric.Tannier@inria.fr

${ }^{1}$ INRIA Rhône-Alpes, 655 avenue de l'Europe, F-38344 Montbonnot, France

Full list of author information is available at the end of the article
}

ancestral gene order based on reconciled phylogenies that account for gene origination, duplication, loss, and transfer.

We propose an algorithm to simultaneously reconstruct all gene organizations along a species phylogeny, minimizing the number of gains and breakages of adjacencies that link consecutive genes on chromosomes. We build upon the dynamic programming principle proposed by Bérard et al. [13] and extend it to consider as input reconciliations containing lateral gene transfer produced by Szöllősi et al. [14].

We implement our algorithm naming the resulting software DeCoLT, in reference to DeCo [13] (Detection of Coevolution) with Lateral Transfers. We examine two datasets of gene trees from a single set of cyanobacteria species. The first set of gene trees is computed from sequence alignements only [15], and the second one is computed by a species tree aware method [16].
C Biomed Central

() 2013 Patterson et al.; licensee BioMed Central Ltd. This is an open access article distributed under the terms of the Creative Commons Attribution License (http://creativecommons.org/licenses/by/2.0), which permits unrestricted use, distribution, and reproduction in any medium, provided the original work is properly cited. 
Our method and the efficiency of the computation is based on the hypothesis that adjacencies evolve independently from each other. While extant genomes consist either of a single or a relatively small number of linear or circular chromosomes, this hypothesis implies that reconstructed ancestral genomes may in theory exhibit more complex arrangements. For example an ancestral gene may be involved in more than two adjacencies, or a large number may have only a single adjacent gene. In the cyanobacteria dataset, extant genomes are all circular, and the ancestral genomes inferred by DeCoLT are also close to being circular with only a few deviations. Most deviations result from the absence of signal to reconstruct genomes in deep ancestors, but some are caused by errors in gene trees, leading to errors in ancestral gene contents. We observe that ancestral genome organizations computed from gene trees that are based on both the species tree and the sequence are closer to being circular than those computed from gene trees based on sequence alone. This argues for the validity of the reconstruction principle we present here and confirms that species tree aware methods produce more accurate gene trees.

\section{Context}

A dated species tree is a rooted binary tree whose leaves are the extant genomes and internal nodes, the ancestral genomes, are totally ordered. This total order, which gives a relative dating to all internal nodes, is supposed to be known. It is, for example, constructed from branch length by a molecular clock technique or from informations on transfers [11]. The time interval between two consecutive internal nodes of a species tree in this total order (one is not necessarily the descendant of the other) defines a time slice. See Figure 1 for an example, where branches leading to $A, B$ or the ancestor of $C$ and $D$ overlap two time slices while the others overlap one. Genomes contain a set of genes and a set of adjacencies, which are pairs of genes, the genes being the two extremities of the adjacency. An adjacency between two genes $a$ and $b$ is noted $a b$ ( $a$ and $b$ always are in the same species, and can be homologous or not). In extant genomes, an adjacency means that two genes are immediately consecutive, with no other gene between them on the chromosome (regardless of their physical distance), so most genes belong to exactly two adjacencies.

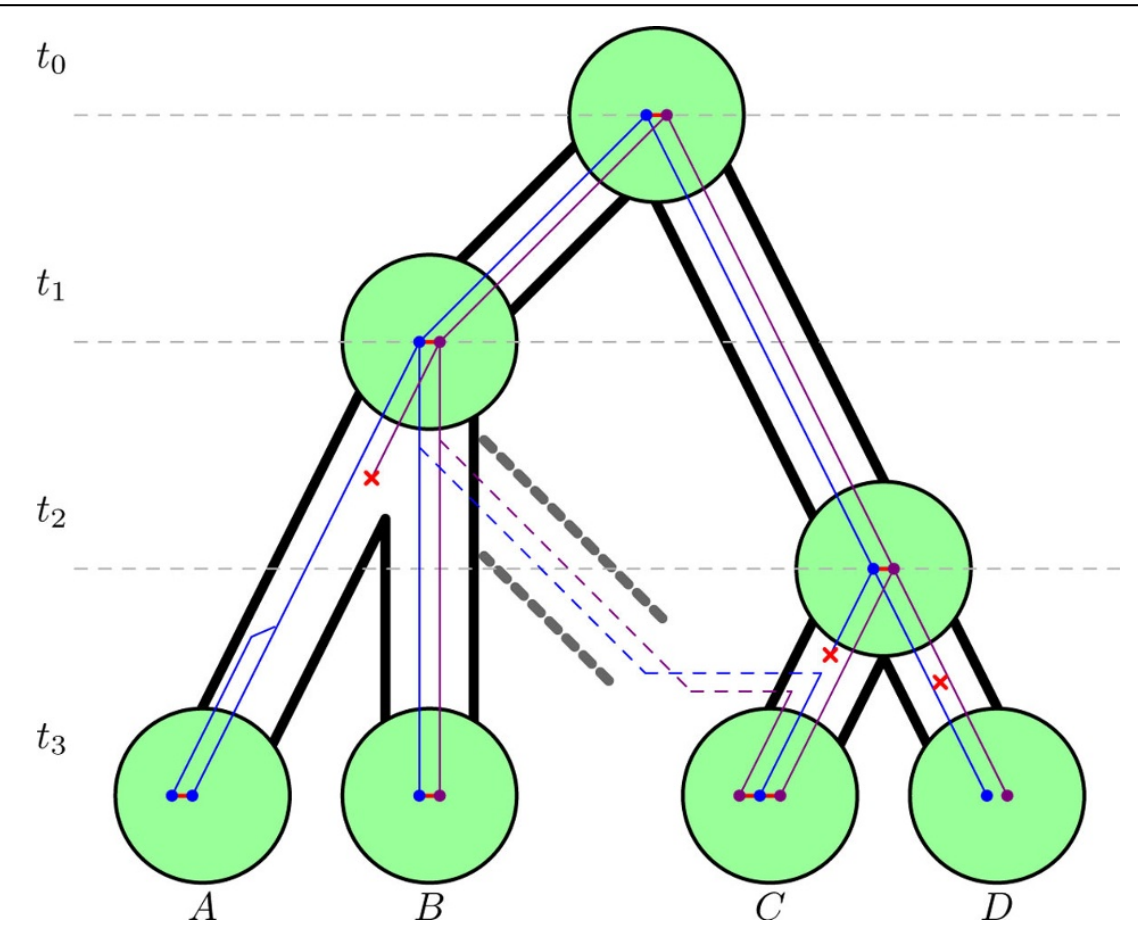

Figure 1 Adjacency evolution. The evolution of an adjacency within a dated species tree, along reconciled gene phylogenies. The gene trees are blue and purple, while red horizontal edges are adjacencies. The time slices $t_{0,} \ldots, t_{3}$ indicate in which order the speciation nodes (big green nodes) occur, and are used to localize genes in the species tree (a branch and a time slice give the coordinates of a gene or an event). Red crosses mean gene losses, for example in the branches leading to $\boldsymbol{A}$ or $\boldsymbol{C}$ (adjacencies are lost when one extremity is lost), or an adjacency breakage, for example in the branch leading to $\boldsymbol{D}$ (gene loss and adjacency breakages are different events, since a gene loss is not a rearrangement while a breakage is, and only rearrangements are counted in the objective function). Here one adjacency is gained in the branch leading to species $\boldsymbol{C}$, one is broken in the branch leading to species $\boldsymbol{D}$, and one is transferred from the branch leading to $\boldsymbol{B}$ to the branch leading to $\boldsymbol{C}$. The transfer implies first a speciation outside the species phylogeny, and then a transfer which can be in another time slice. A tandem duplication in the branch leading to $\boldsymbol{A}$ gives a new adjacency between the two copies. 
Genes of all genomes are partitioned into homologous families, and each family is organized in a gene tree, which is a rooted tree whose nodes are the genes, describing the pattern of descent within a family. Gene trees are reconciled with the species tree, which means that nodes and branches of gene trees are annotated to account for the particular history of the gene family. Possible events are origination (of the gene in the species tree), speciation (genes follow the species diversification), duplication, transfer, or loss. Transfers are the acquisition of a gene by a genome in the species tree from a genome outside the species tree. Indeed genes at the origin of transfers almost always belong to unsampled or extinct species [14]. That is why speciation does not only happen at the nodes of the species tree. A gene can also leave the species tree by speciation from a species tree branch, and be transferred back later (see Figure 1 for an example). But we assume that genes do not diversify (either by speciation or by duplication) outside the species tree. Every event on a gene tree is associated to a branch and a time slice of the species tree.

The input to our method is a dated species tree, a set of reconciled gene trees and the set of extant adjacencies. Reconciled gene trees yield ancestral genes (dots inside green circles in Figure 1). The problem will be to construct the ancestral adjacencies, given this input. In practice the input is provided by methods and software described in Szöllősi et al.'s trilogy $[11,14,16]$. The first paper of this trilogy explains how to find the dated species tree, the second one how to reconcile gene trees taking extinct or unsampled species into account, and the third one how to reconstruct species tree aware gene trees.

We construct ancestral adjacencies in a manner that minimizes the number of rearrangements along the species phylogeny. This number is computed as the number of gains and breakages of adjacencies necessary to explain all extant adjacencies. For example, if we infer no ancestral adjacencies at all, then the value of this objective function is proportional to the number of extant adjacencies, because all of them are gained independently. If we propose an adjacency in an ancestral genome which is a common ancestor of a set of adjacencies, then the value decreases because all adjacencies in that set are explained by a unique gain (see Figure 1, where three extant adjacencies can be explained with two gains and a breakage).

We then have to describe how an ancestral adjacency propagates within reconciled gene trees so that it can be recognized as an ancestor of an extant one.

\section{Propagation rules}

The two extremities of an adjacency are clearly always in the same species (extant or ancestral) at the same time slice. If there is an adjacency between two ancestral genes $a$ and $b$, it is propagated to the descendants, in the absence of rearrangements, following the rules described in Figure 2, according to the events happening to genes $a$ and $b$.

A history is a set of ancestral and extant adjacencies. In a history, any adjacency which does not have a parent identified by the propagation rules yields an adjacency gain. A breakage is inferred when an adjacency is present in the history but one of its descendants according to the propagation rules is not. The cost of a history is the number of gains and breakages it yields.

\section{Algorithm}

We compute a minimum cost history by writing a dynamic programming algorithm following the propagation rules and adding adjacency gains and breakages with costs that are considered in the optimization. In order to solve a more general problem and to present the recurrence formulas more clearly, gains and breakages are assigned a cost, which could be different, and we minimize on the number of events weighted by their cost. In practice we always use the algorithm with equal costs, thus minimizing the sum of the number of gains and breakages.

\section{Classes of adjacencies}

Two adjacencies are homologous with respect to a particular history if they descend from a common ancestor following the propagation rules. Homology of adjacencies is an equivalence relation. We first state a necessary condition for a set of adjacencies to be homologous in order to restrict the search space for homology.

Two extant adjacencies $a_{1} b_{1}$ and $a_{2} b_{2}$ are possibly homologous if there are two ancestral genes $a$ and $b$ of an ancestral genome $G$, such that $a$ (resp., $b$ ) is an ancestor of $a_{1}$ and $a_{2}$ (resp., $b_{1}$ and $b_{2}$ ). This simply tells us that in order to find a common ancestor of two adjacencies, there has to exist two genes being the extremities of this adjacency. So if two adjacencies are homologous with respect to a particular history then they are possibly homologous (the definition of possible homology is independent from any history). Possible homology, defined on two adjacencies, is obviously a symmetric and reflexive relation. It is also transitive, partitioning the set of extant adjacencies into equivalence classes.

Consequently, homology can be searched within a class. For each class $\left\{a_{1} b_{1}, \ldots, a_{k} b_{k}\right\}$, there are two genes $a$ and $b$, such that $a$ (resp. $b$ ) is an ancestor of all $a_{i}$ (resp., $\left.b_{i}\right)$. Among the possible such genes $a$ and $b$ for a class, we call the highest distinct ones the roots of that class. We then work with the disjoint subtrees rooted by $a$ and $b$, and find a history following the propagation rules for all adjacencies whose extremities are descendants of $a$ and $b$. Hence, it is sufficient to search within pairs of trees to construct a history. 


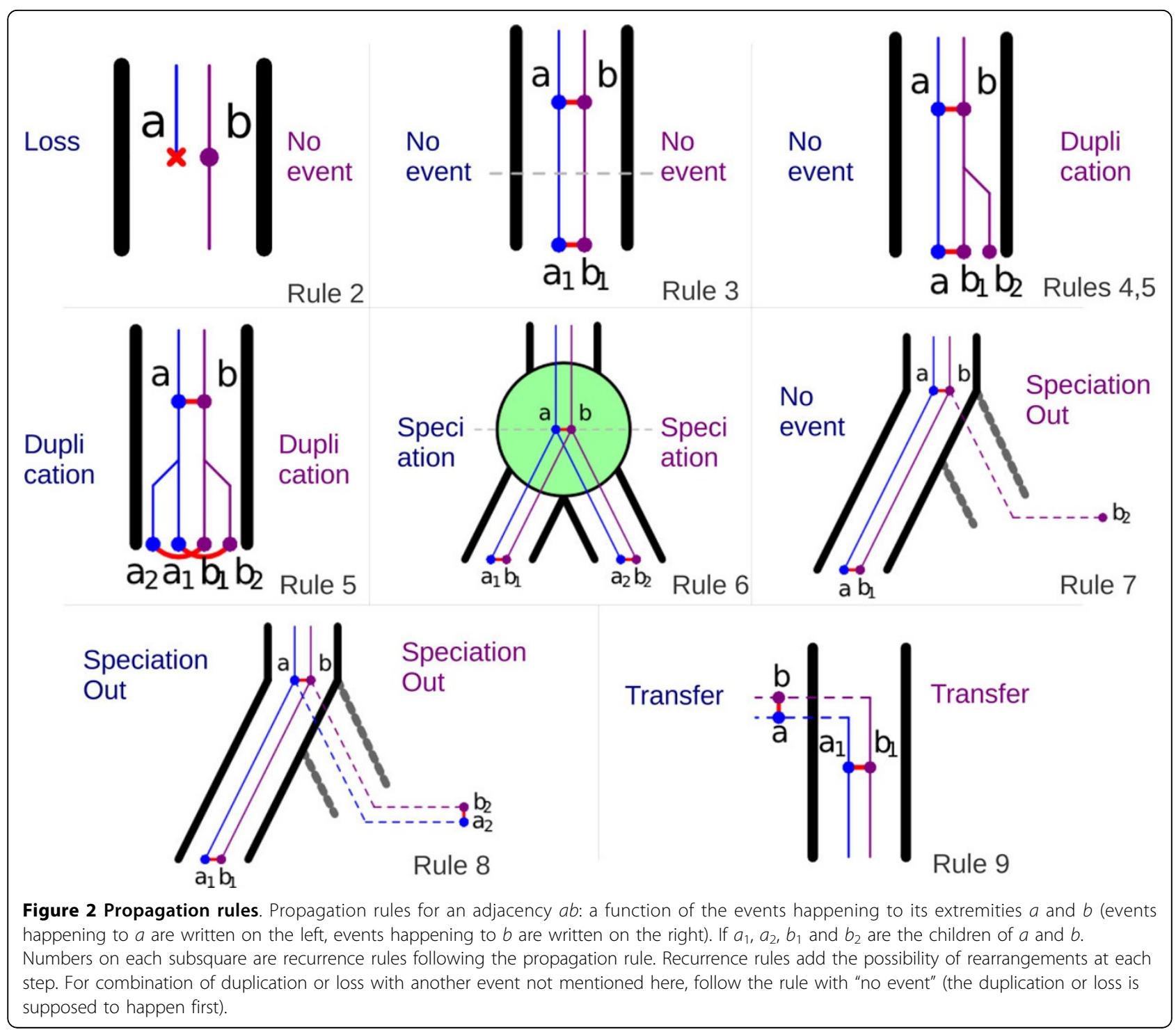

\section{Recurrence formulas within one class}

For any two gene tree nodes $a$ and $b$, for which $s(a)=s(b)$, let $c_{1}(a, b)$ be the minimum cost of a history for the two gene subtrees rooted at $a$ and $b$, assuming there is an adjacency between $a$ and $b$, and let $c_{0}(a, b)$ be the minimum cost of a history for two gene subtrees rooted at $a$ and $b$, assuming there is no adjacency between $a$ and $b$. The values of $c_{1}(a, b)$ and $c_{0}(a, b)$ are recursively computed, according to the events annotating $a$ and $b$.

The principle of these formulas is to describe the propagation rules and add to them the possibility of rearrangements (gains and breakages of adjacencies). We compute $c_{1}(a, b)$ and $c_{0}(a, b)$ as a function of $c_{1}$ and $c_{0}$ for the children of $a$ and $b$. So we have to consider all combinations of presence or absence of adjacencies between the children. That is why some cases may imply up to 16 different subcases because of the symmetry of the children of $a$ and $b$, if they are in the same species.

Given a node $u(u=a$ or $u=b), u_{1}$ is the first (or only child of $u$ in the case that $u$ has only one child), while $u_{2}$ is the second child. We write $E(u)$ to denote the event at node $u$, where $E(u)=$ Extant when $u$ is a leaf of a gene tree corresponding to an extant gene.

Case $1 E(a)=$ Extant and $E(b)=$ Extant (both nodes are leaves).

In this case, if $a b$ is an adjacency then $c_{1}(a, b)=0$ and $c_{0}(a, b)=\infty$, else $c_{1}(a, b)=\infty$ and $c_{0}(a, b)=0$.

Case $2 E(a)=$ GeneLoss (one of the genes is lost, any event may happen to the other).

In this case $c_{1}(a, b)=0$ and $c_{0}(a, b)=0$.

Case $3 E(a)=$ NoEvent and $E(b)=$ NoEvent (both gene trees are changing time slice without any event). 
In this case $c_{1}(a, b)=\min \left\{c_{1}\left(a_{1}, b_{1}\right), c_{0}\left(a_{1}, b_{1}\right)+C\right.$ (Break) $\}$ and $c_{0}(a, b)=\min \left\{c_{0}\left(a_{1}, b_{1}\right), c_{1}\left(a_{1}, b_{1}\right)+C(\right.$ Gain $\left.)\right\}$.

Case $4 E(a) \in\{$ Extant, NoEvent, Speciation, SpeciationOut $\}$ and $E(b)=$ GeneDuplication

In this case we suppose that the duplication of $b$ happens before any event in the gene tree containing $a$. Here, $c_{1}(a, b)=\mathbf{D} \mathbf{1}$, and $c_{0}(a, b)=\mathbf{D} \mathbf{0}$, where

$$
\text { D1 }=\min \left\{\begin{array}{l}
c_{1}\left(a, b_{1}\right)+c_{0}\left(a, b_{2}\right), \\
c_{0}\left(a, b_{1}\right)+c_{1}\left(a, b_{2}\right), \\
c_{1}\left(a, b_{1}\right)+c_{1}\left(a, b_{2}\right)+C(\text { Gain }), \\
c_{0}\left(a, b_{1}\right)+c_{0}\left(a, b_{2}\right)+C(\text { Break })
\end{array} \quad \text { D0 }=\min \left\{\begin{array}{l}
c_{0}\left(a, b_{1}\right)+c_{0}\left(a, b_{2}\right), \\
c_{0}\left(a, b_{1}\right)+c_{1}\left(a, b_{2}\right)+C(\text { Gain }), \\
c_{1}\left(a, b_{1}\right)+c_{0}\left(a, b_{2}\right)+C(\text { Gain }), \\
c_{1}\left(a, b_{1}\right)+c_{1}\left(a, b_{2}\right)+2 * C(\text { Gain })
\end{array}\right.\right.
$$

Case $5 E(a)=$ GeneDuplication and $E(b)=$ GeneDuplication.

In this case $c_{1}(a, b)=\min (\mathbf{D} 1, \mathbf{D} 12, \mathbf{D} 12)$ where

D1 (defined in Case 4) is the cost in the case where the $a$ duplication comes first,

D2 is the cost in the case where the $a$ duplication comes first, and

$$
\mathbf{D} 2=\min \left\{\begin{array}{l}
c_{1}\left(a_{1}, b\right)+c_{0}\left(a_{2}, b\right) \\
c_{0}\left(a_{1}, b\right)+c_{1}\left(a_{2}, b\right) \\
c_{1}\left(a_{1}, b\right)+c_{1}\left(a_{2}, b\right)+C(\text { Gain }), \\
c_{0}\left(a_{1}, b\right)+c_{0}\left(a_{2}, b\right)+C(\text { Break })
\end{array}\right.
$$

D12 is the cost in the case where the $a$ and $b$ duplications are simultaneous, where D12 $=\min$ (over all 16 of the following cases):

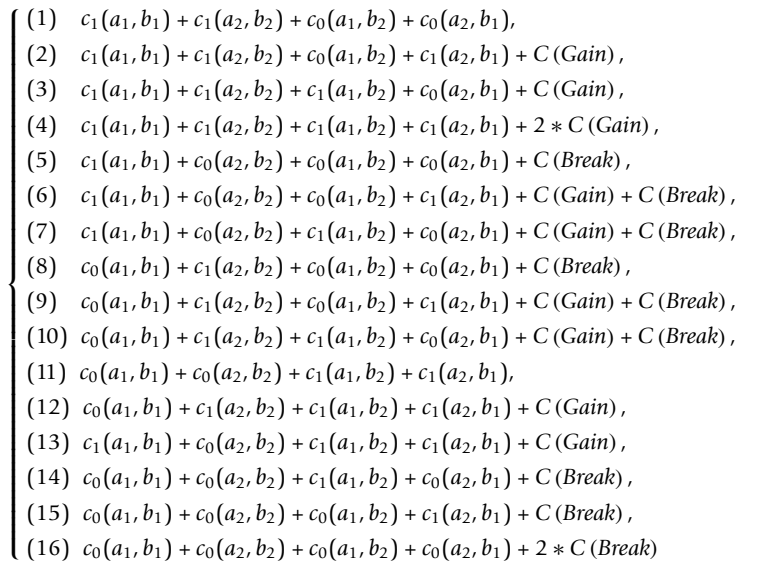

The multiplication of the cases come from the symmetry of the children of both $a$ and $b$ (for example, (1) is the symmetric subcase of (11)). As there are four children, there are four possible pairings between children from $a$ and $b$, so we examine all combinations, that is, $2^{4}=16$.

Here, $c_{0}(a, b)=$ D00, where

$$
\mathbf{D 0 0}=\min \left\{\begin{array}{l}
\text { Do } \\
c_{0}\left(a_{1}, b\right)+c_{0}\left(a_{2}, b\right) \\
c_{0}\left(a_{1}, b\right)+c_{1}\left(a_{2}, b\right)+C(\text { Gain }) \\
c_{0}\left(a_{1}, b\right)+c_{0}\left(a_{2}, b\right)+C(\text { Gain }) \\
c_{1}\left(a_{1}, b\right)+c_{1}\left(a_{2}, b\right)+2 * C(\text { Gain })
\end{array}\right.
$$

Case $6 E(a)=$ Speciation and $E(b)=$ Speciation .

We assume without loss of generality that $s\left(a_{1}\right)=s\left(b_{1}\right)$ and $s\left(a_{2}\right)=s\left(b_{2}\right)$. Here, $c_{1}(a, b)=\mathbf{S 1}$ and $c_{0}(a, b)=\mathbf{S} \mathbf{0}$, where

$$
\text { S1 }=\min \left\{\begin{array}{l}
c_{1}\left(a_{1}, b_{1}\right)+c_{1}\left(a_{2}, b_{2}\right), \\
c_{1}\left(a_{1}, b_{1}\right)+c_{0}\left(a_{2}, b_{2}\right)+C(\text { Break }), \\
c_{0}\left(a_{1}, b_{1}\right)+c_{1}\left(a_{2}, b_{2}\right)+C(\text { Break }), \\
c_{0}\left(a_{1}, b_{1}\right)+c_{0}\left(a_{2}, b_{2}\right)+2 * C(\text { Break })
\end{array} \quad \text { So }=\min \left\{\begin{array}{l}
c_{0}\left(a_{1}, b_{1}\right)+c_{0}\left(a_{2}, b_{2}\right), \\
c_{1}\left(a_{1}, b_{1}\right)+c_{0}\left(a_{2}, b_{2}\right)+C(\text { Gain }), \\
c_{0}\left(a_{1}, b_{1}\right)+c_{1}\left(a_{2}, b_{2}\right)+C(\text { Gain }), \\
c_{1}\left(a_{1}, b_{1}\right)+c_{1}\left(a_{2}, b_{2}\right)+2 * C(\text { Gain })
\end{array}\right.\right.
$$

Case $7 E(a) \in\{$ Extant, NoEvent, Speciation $\}$ and $E(b)$ = SpeciationOut.

In this case $c_{1}(a, b)=c_{1}\left(a, b_{1}\right)$ and $c_{0}(a, b)=c_{0}\left(a, b_{1}\right)$.

Case $8 E(a)=$ SpeciationOut and $E(b)=$ SpeciationOut .

We assume without loss of generality that $a_{1}$ (resp., $b_{1}$ ) is the child that remains inside the species tree, while $a_{2}$ (resp., $b_{2}$ ) is the child that leaves the tree. In this case, $c_{1}(a, b)=c_{1}\left(a_{1}, b_{1}\right)+\min \left\{c_{1}\left(a_{2}, b_{2}\right), c_{0}\left(a_{2}, b_{2}\right)\right.$ $+C($ Break $)\}$ and $c_{0}(a, b)=c_{0}\left(a_{1}, b_{1}\right)+\min \left\{c_{0}\left(a_{2}, b_{2}\right), c_{1}\right.$ $\left(a_{2}, b_{2}\right)+C($ Gain $\left.)\right\}$.

Case $9 E(a)=$ Transfer and $E(b)=$ Transfer .

In this case, $c_{1}(a, b)=\min \left\{c_{1}\left(a_{1}, b_{1}\right), c_{0}\left(a_{1}, b_{1}\right)+C\right.$ $($ Break $)\}$ and $c_{0}(a, b)=\min \left\{c_{0}\left(a_{1}, b_{1}\right), c_{1}\left(a_{1}, b_{1}\right)+C\right.$ (Gain)\}.

Observe that if $E(a)=$ Transfer and $E(b) \notin\{$ Transfer, GeneLoss\}, or if $a$ and $b$ do not have a SpeciationOut ancestor in the same species, then $(a, b)$ will form the root of an additional equivalence class. Indeed in this case $c_{1}(a, b)$ and $c_{0}(a, b)$ cannot be recursively called.

\section{Backtracking Procedure}

First, the dynamic programming matrix $M[a, b]$ containing a cell for each pair $(a, b)$ of nodes in the respective gene trees is created by following the recurrence rules for each equivalence class.

Then, after applying the classical backtracking procedure on $M$, the optimal (minimum cost) history is then obtained by choosing the minimum among $c_{1}+C$ (Gain) and $c_{0}$ for the roots of all classes.

\section{Complexity}

Let $m$ be the number of gene trees, $n$ be the maximum number of genes in a gene tree, and $s$ be the number of species. There are $s-1$ time slices, so every branch of a gene tree may be subdivided as many as $s-1 \leq n$ times, which gives at most $n^{2}$ events (most of them are NoEvent events) in a given tree. The number of equivalence classes is $O\left(m^{2}\right)$, and hence there are $O\left(m^{2} n^{4}\right)$ comparisons computed during the initial construction of dynamic programming matrix $M$.

In practice, the number of equivalence classes is much smaller, closer to $m$ than $O\left(m^{2}\right)$, and the majority of the $O$ $\left(n^{2}\right)$ events each tree are NoEvent events. On the cyanobacteria dataset of $(m=) 1099$ families from $(n=) 36$ genomes, our implementation, DeCoLT, of this algorithm constructed the adjacencies in under 3 hours on a standard desktop computer. 


\section{Cyanobacteria ancestral genomes}

The algorithm has been implemented and run on two datasets. They both have the same species tree (depicted in Figure 3), on the same set of 36 extant genomes from cyanobacteria and the same extant adjacencies.

They differ by their set of gene trees. One of them is the sequence trees, which are maximum likelihood trees constructed from a model of sequence evolution using multiple alignments of protein sequences of extant genes from each family, taken from [11].

The other is the $A L E$ trees, which are maximum likelihood trees constructed from a model of sequence evolution in conjunction with a birth and death branching model to account for origination, duplication, transfer and loss, taken from [16]. As transfers are very likely to involve lineages outside any given phylogeny [14], reconciled trees have nodes leaving the species tree (SpeciationOut) and nodes transferring to the species tree (Transfer). Note that the reconciliations may contain "speciation outside" nodes, which mean diversification of a gene outside the species tree, and our algorithm do not handle these nodes for the moment. In order to handle these datasets we simply removed these nodes, which has the effect of cutting some trees into pieces.

For both datasets, ancestral adjacencies were computed using DeCoLT. The degree of each ancestral gene (the number of adjacencies it belongs to) was computed, and we then plotted the proportion of ancestral genes having degree $k$ for $k$ between 0 and 6 (Figure 4).

There are almost no genes with degree larger than 2 in either dataset. The proportion of genes with degree 2 increases from $17 \%$ for sequence trees to $31 \%$ for ALE trees. This means that we: (i) accurately reconstruct ancestral adjacencies because they all have a circular structure; and (ii) the quality of gene trees nearly doubles the resolution of ancestral genomes. Finally, having only $31 \%$ of ancestral genes with degree two means that a large part of the gene order signal is lost in this very deep branch. However, this is not the case for ancestral genomes. The size of the nodes on Figure 3 indicates the ratio between the number of adjacencies and the number of genes in each genome (the ideal ratio is 1 ). In the Prochlorococcus clade, over $80 \%$ of the genomes are reconstructed whereas it drops to nearly $0 \%$ in deeper nodes.

We found that 64 clusters of genes were co-transferred: transferred adjacencies were detected, as well as 28 clusters of co-duplicated genes during the evolution of cyanobacteria. Most are simply pairs of genes, but there is a cluster of 4 co-transferred genes, four clusters of 3 co-transferred genes, and two clusters of 3 co-duplicated genes.

\section{Discussion}

The optimizing property of the algorithm follows from the exact translation of the propagation rules into the recurrence formulas, adding all possibilities of rearrangements in each step. It is a generalization of the reconstruction of discrete ancestral characters solved by Sankoff-Rousseau type algorithms [17]. To see this, one can observe that our framework is strictly equivalent to the Sankoff-Rousseau algorithm [17] in the case where there are no events in the trees.

Further improvements in the method would consist in adding the possibility of homolog replacement when a gene is transferred: for the moment any transfer yields rearrangements whereas some genes might replace an homologous one, keeping the gene order unchanged. We could also think of avoiding rearrangements caused

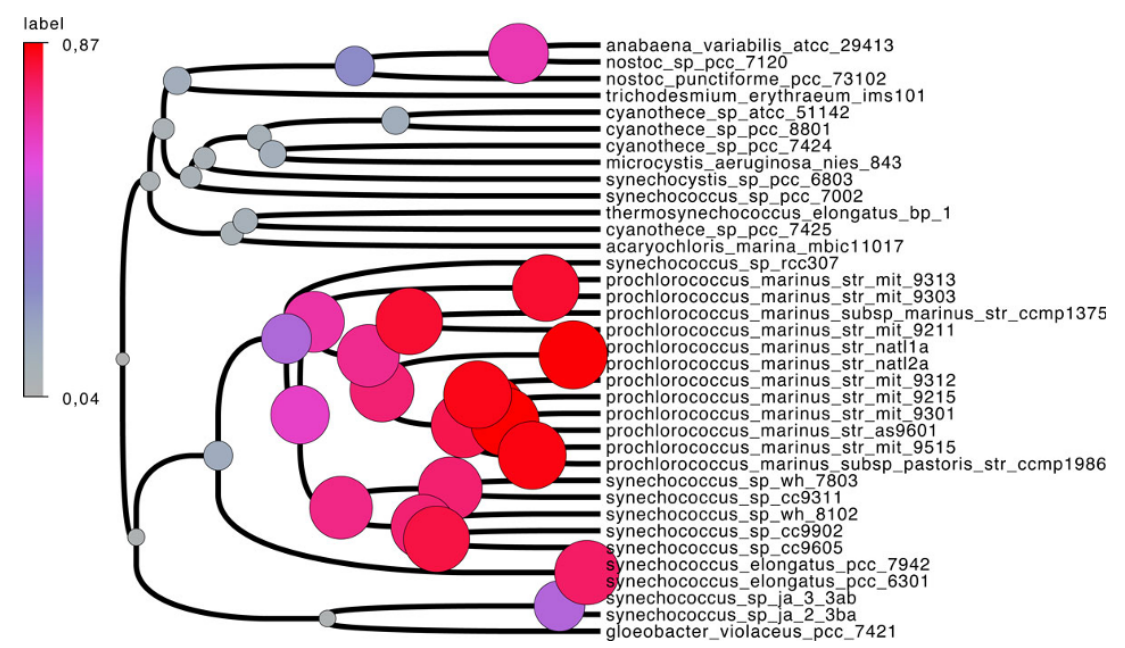

Figure 3 Cyanobacteria phylogeny. Cyanobacteria dated species tree. The size (area) and colours of internal nodes is the ratio of the number of adjacencies over the number of genes in every ancestral species. 


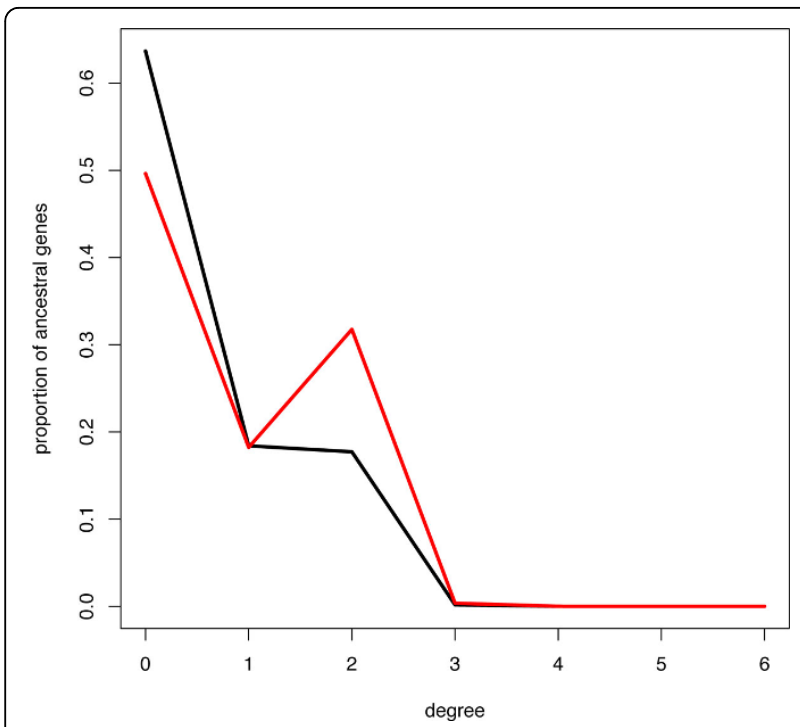

Figure 4 Circularity of ancestral genomes. On the $x$ axis is the degree of a gene, that is, the number of adjacencies it belongs to, and on the $y$ axis there is the proportion of genes with this degree. In black, there are the values for the sequence trees and in red for ALE trees.

by origination and losses of genes, which, for the moment, necessarily yield several adjacency gains and losses.

Future work will also consist in deriving function information from co-transfers, and trying the same principles on other kinds of relations than adjacencies, starting for example from the relation between domains forming the same gene.

\section{Competing interests}

The authors declare that they have no competing interests.

\section{Authors' contributions}

MP, GS, VD and ET devised the algorithm and wrote the paper. MP programmed the software.

\section{Acknowledgements}

The authors would like to thank Sèverine Bérard and Thomas Bigot for advice on the implementation aspects of DeCoLT. MP is funded by a Marie Curie Fellowship from the Alain Bensoussan program of ERCIM and and GS is funded by the Marie Curie Fellowship 253642 "Geneforest", and the Albert Szent-Györgyi Scholarship A1-SZGYA-FOK-13-0005. This work is funded by the Agence Nationale pour la Recherche, Ancestrome project ANR-10-BINF01-01.

This article has been published as part of BMC Bioinformatics Volume 14 Supplement 15, 2013: Proceedings from the Eleventh Annual Research in Computational Molecular Biology (RECOMB) Satellite Workshop on Comparative Genomics. The full contents of the supplement are available online at http://www.biomedcentral.com/bmcbioinformatics/supplements/ $14 / S 15$.

\section{Authors' details}

${ }^{\prime}$ INRIA Rhône-Alpes, 655 avenue de l'Europe, F-38344 Montbonnot, France. 2Laboratoire de Biométrie et Biologie Évolutive, CNRS and Université de Lyon 1, 43 boulevard du 11 novembre 1918, F-69622 Villeurbanne, France. ${ }^{3}$ Centrum Wiskunde \& Informatica, Science Park 123, 1098 XG, Amsterdam, The Netherlands. "ELTE-MTA "Lendület" Biophysics Research Group 1117 Bp., Pázmány P. stny. 1A., Budapest, Hungary.

Published: 15 October 2013

\section{References}

1. Sturtevant A, Dobzhansky T: Inversions in the third chromosome of wild races of Drosophila pseudoobscura, and their use in the study of the history of the species. Proc Natl Acad Sci USA 1936, 22:448-450.

2. Sturtevant A, Tan C: The comparative genetics of Drosophila Pseudoobscura and Drosophila Melanogaster. Journal of genetics 1937, 34:415-432.

3. Fertin G, Labarre A, Rusu I, Tannier E, Vialette S: Combinatorics of genome rearrangements. MIT press 2009.

4. Lin Y, Hu F, Tang J, Moret B: Maximum Likelihood Phylogenetic Reconstruction from High Resolution Whole-Genome Data and a Tree of 68 Eukaryotes. Pacific Symposium on Biocomputing 2013.

5. Zuckerkandl $E$, Pauling L: Molecules as documents of evolutionary history. J Theor Biol 1965, 8(2):357-366.

6. Felsenstein J: Inferring phylogenies Sinauer Associates, Inc; 2004.

7. Page R: Maps between Trees and Cladistic Analysis of Historical Associations among Genes, Organisms, and Areas. Systematic Biology 1994, 43:58-77.

8. Maddison WP: Gene Trees in Species Trees. Systematic Biology 1998, 46:523-536.

9. Arvestad L, Berglund AC, Lagergren J, Sennblad B: Bayesian gene/species tree reconciliation and orthology analysis using MCMC. Bioinformatics 2003, 19:17-15.

10. Doyon JP, Ranwez V, Daubin V, Berry V: Models, algorithms and programs for phylogeny reconciliation. Brief Bioinform 2011, 12(5):392-400[http://dx. doi.org/10.1093/bib/bbr045].

11. Szöllősi GJ, Boussau B, Abby SS, Tannier E, Daubin V: Phylogenetic modeling of lateral gene transfer reconstructs the pattern and relative timing of speciations. Proc Natl Acad Sci USA 2012, 109(43):17513-17518 [http://dx.doi.org/10.1073/pnas.1202997109].

12. Sankoff D, El-Mabrouk N: Duplication, rearrangement and reconciliation. In Comparative Genomics: Empirical and Analytical Approaches to Gene Order Dynamics, Map alignment and the Evolution of Gene Families, Volume 1 of Computational Biology. Kluwer Academic Press;Sankoff D, Nadeau JH 2000.

13. Bérard S, Gallien C, Boussau B, Szöllősi G, Daubin V, Tannier E: Evolution of gene neighborhood within reconciled phylogenies. Bioinformatics 2012, 28:i382-i388.

14. Szöllősi GJ, Tannier E, Lartillot N, Daubin V: Lateral Gene Transfer from the Dead. Syst Biol 2013, 62:386-397[http://dx.doi.org/10.1093/sysbio/syt003].

15. Guindon S, Dufayard JF, Lefort V, Anisimova M, Hordijk W, Gascuel O: New algorithms and methods to estimate maximum-likelihood phylogenies: assessing the performance of PhyML 3.0. Syst Biol 2010, 59(3):307-321 [http://dx.doi.org/10.1093/sysbio/syq010]1.

16. Szöllősi GJ, Rosikiewicz W, Bousseau B, Tannier E, Daubin V: Efficient Exploration of the Space of Reconciled Gene Trees. 2013, [Accepted by Sys Biol].

17. Sankoff D: Minimal Mutation Trees of Sequences. SIAM Journal on Applied Mathematics 1975, 28:35.

\section{doi:10.1186/1471-2105-14-S15-S4}

Cite this article as: Patterson et al:: Lateral gene transfer, rearrangement, reconciliation. BMC Bioinformatics 2013 14(Suppl 15):S4.

\section{Submit your next manuscript to BioMed Central and take full advantage of:}

- Convenient online submission

- Thorough peer review

- No space constraints or color figure charges

- Immediate publication on acceptance

- Inclusion in PubMed, CAS, Scopus and Google Scholar

- Research which is freely available for redistribution 